\title{
A novel method of treating isolated unicondylar fracture of the head of the proximal phalanx: A case report
}

\author{
Aysha Rajeev, John Harrison
}

\begin{abstract}
Introduction: The phalangeal fractures are common hand injuries. The unicondylar fractures of proximal phalanx are unique. They need prompt and accurate treatment to have a good functional outcome. We present a technique for managing a volar displaced (type 4) unicondylar fracture of the proximal phalanx. This provides accurate reduction and stabilization until fracture union, with minimal soft tissue tethering to allow early movement. Case Report: A 26-years-old male sustained an injury to the right index finger while playing cricket. He attended fracture clinic complaining of pain and difficulty in moving the proximal interphalangeal (PIP) joint of the index finger. The X-ray showed a unicondylar fracture of the proximal phalanx in the coronal plane, with a displaced volar fragment. The patient was treated with open reduction and internal fixation with a single Kirschner wire (K-wire) passed from volar to dorsal aspect through the fragment. The patient commenced hand physiotherapy straight away and regained full range of movements. Conclusion: We report the technique of fixing the displaced unicondylar fractures of the proximal phalanx using single K-wire passed from the volar to dorsal aspect
\end{abstract}

Aysha Rajeev ${ }^{1}$, John Harrison ${ }^{2}$

Affiliations: ${ }^{1} \mathrm{FRCS}$, Associate Specialist,Trauma and Orthopaedics, Queen Elizabeth Hospital, Gateshead, UK; ${ }^{2}$ FRCS, Consultant, Trauma and Orthopaedics, Queen Elizabeth Hospital, Gateshead, UK.

Corresponding Author: Aysha S Rajeev, 25,Fatfield Park, Washington, Tyne and Wear, NE38 8BW; Ph: 00447414262665; Fax: 00441914453874; Email: asrajeev18@gmail.com

Received: 23 June 2012

Accepted: 01 September 2012

Published: 01 January 2013 after open reduction of the fragment through a volar approach. This technique will allow early mobilization with very minimal soft tissue dissection thus preventing stiffness.

Keywords: Unicondylar, Proximal phalanx, Type 4 fracture

$$
* * * * * * * * *
$$

Rajeev A, Harrison J. A novel method of treating isolated unicondylar fracture of the head of the proximal phalanx: A case report. International Journal of Case Reports and Images 2013;4(1):41-45.

$* * * * * * * * *$

doi:10.5348/ijcri-2013-01-256-CR-10

\section{INTRODUCTION}

Unicondylar fractures of the phalangeal head are relatively common injuries. They are more common in young, active patients and define a specific fracture pattern [1]. These are intra-articular fractures and four types are described [2]. Class 1 and 2 occur between the condyles (intercondylar) leading to angular deformity of the digit if displaced. Class 3 is a dorsal fragment in the coronal plane. Class 4 fractures are less common and is a volar fragment. If displaced these fractures require operative management. Intercondylar fractures may be treated successfully with closed reduction and percutaneous transverse fixation. Coronal plane fractures may require open reduction and any metalwork must not be left in the joint [3]. Accurate reduction and stabilization, allowing early motion are the key aims for achieving good results with all types of unicondylar fractures of the proximal phalanx. When stabilization is necessary, soft tissue trauma should be minimized to lessen the risk of scar formation and consequent stiffness at the proximal interphalangeal 
joint. Stiffness is the most frequent and serious reported complication of unicondylar fractures [4].

\section{CASE REPORT}

A 26-years-old male with right dominant hand was seen in the fracture clinic following an injury to his right index finger. $\mathrm{He}$ worked as a computer sales representative. He sustained a hyperextension injury to his finger while playing cricket when the cricket ball hit his index finger tip. He complained about pain and difficulty in moving the proximal interphalangeal (PIP) joint. He was otherwise fit and well.

On examination, there was tenderness in the PIP joint. With the finger extended there was no angular deformity. Movement at the PIP joint was limited to O-40 degrees of flexion. Metacarpophalangeal (MCP) and distal interphalengeal (DIP) joint movements were full. The X-ray showed a unicondylar fracture of the proximal phalanx in the coronal plane (Figure 1), with a displaced volar fragment. The patient was offered surgery after explaining the risk of stiffness and malunion.

Under general anaesthetic and tourniquet control the PIP joint was exposed through a Brunner's incision (Figure 2). The A3 pulley was released on the ulnar side. The condylar fragment was reduced and held with a Kirschner wire (K-wire) passed from volar to dorsal through the fragment. The K-wire driver was then moved to the leading end of the wire where it protruded through the skin and was then pulled through so the trailing end of the K-wire was just under the articular surface (Figure 3). The reduction was checked with image intensifier, and full flexion of the PIP joint was achieved.

The patient was allowed to commence finger flexion from the first post-operative day. The patient was followed up in the clinic after two weeks for wound inspection and check X-rays were satisfactory (Figure 4). The patient was able to flex the PIP joint at this stage to $80^{\circ}$. The K-wire was removed four weeks after surgery and the patient was referred for futher intensive physiotherapy. He regained full range of movements of the PIP joint after eight weeks. The X-ray showed that the fracture had healed in an anatomical position (Figure 5). He was discharged from the outpatient clinic.

\section{DISCUSSION}

Various methods of open reduction and internal fixation of unicondylar fractures of the proximal phalanx have been described in literature. London et al. noted that some of these fractures were stable so that they can be treated with splints and early mobilization, whereas others needed open reduction and fixation [4]. $\mathrm{He}$ also proposed a classification system for these fractures. McCue et al. in a review of twenty cases reported that there was no predominance of either

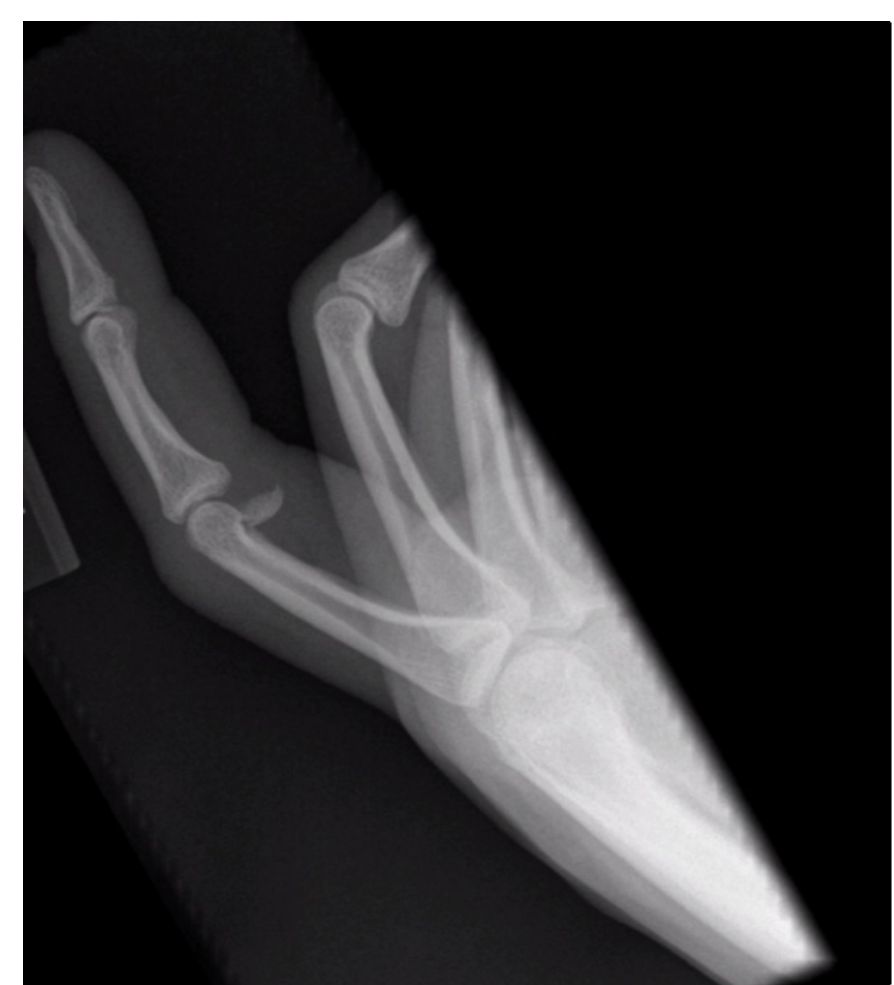

Figure 1: X-ray showing class 4 unicondylar fracture with volar displacement.

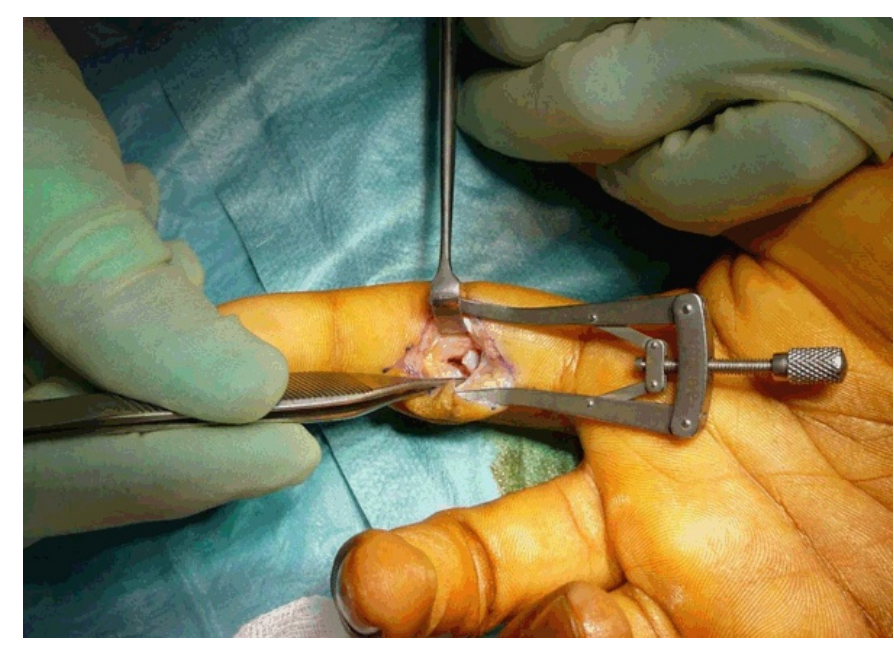

Figure 2: Intra-operative picture showing displaced volar fragment of proximal phalanx.

radial or ulnar condyle involvement in these fractures [5]. They described that unicondylar fractures of PIP joint were most commonly associated with sports injuries. Their treatment protocol was to fix all twenty cases with two Kirschner wires which regained an average of $93^{\circ}$ of PIP joint movements. The mechanism of distal unicondylar fractures of the proximal and middle phalanx were caused by a trochlear shear rather than compression as described by Soeur et al. [6].

Ramos et al. developed a protocol for isolated unicondylar fracture of the head of the proximal phalanx which included surgical fixation using lag screw 


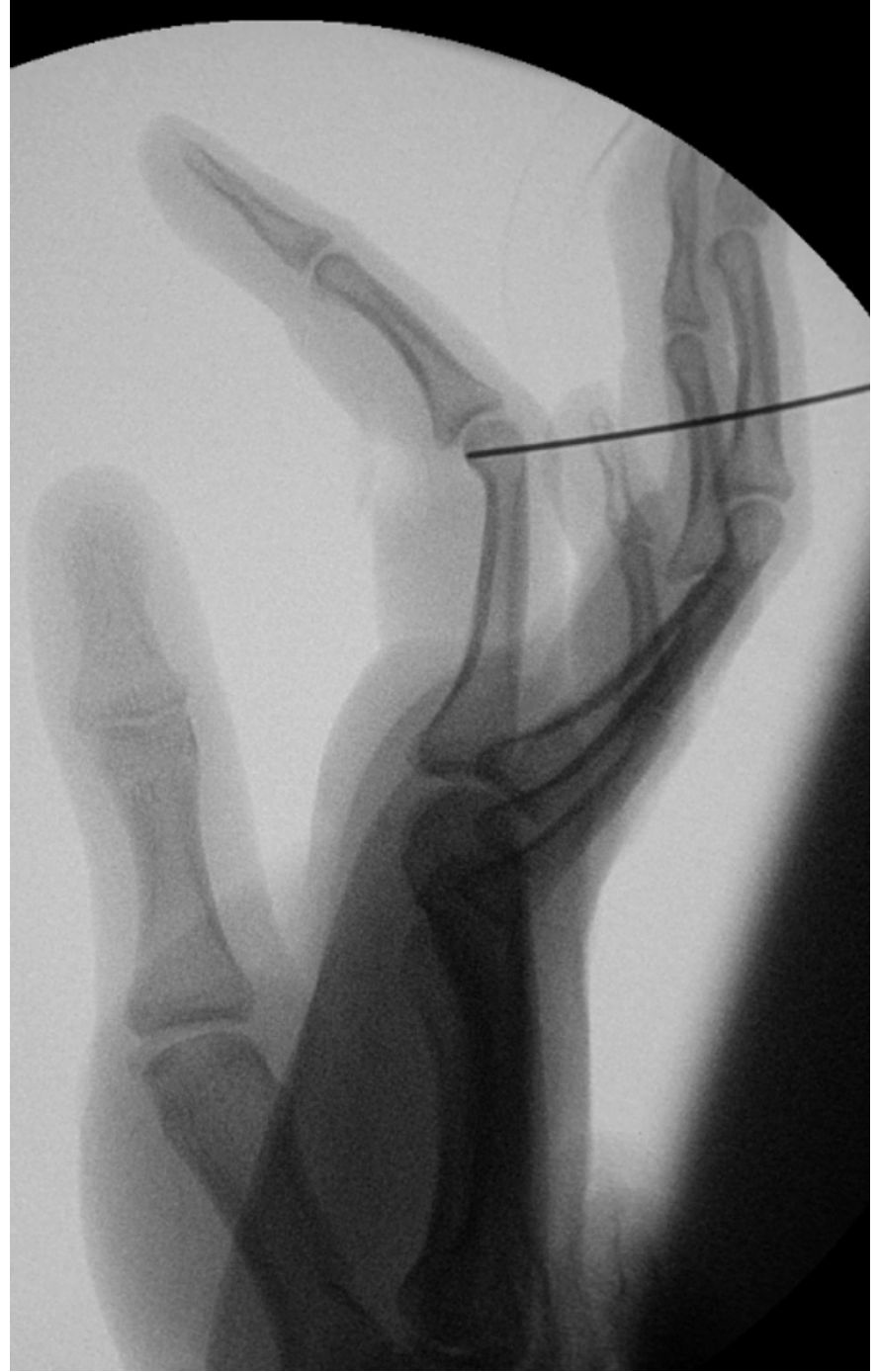

Figure 3: Intra-operative radiograph showing reduction of fracture with the K-wire passed from dorsal aspect.

and immediate mobilization by use of a continous passive motion and controlled active motion [7]. They used specially designed splints and Coban wrap to control the position of the digit during the first six months.

Henry et al. described a wide array of treatment options for these type of fractures of proximal phalanx, including Kirschner wires screws and plate fixation [8]. He stated that early closed reduction is successful for unicondylar fractures of the head of the proximal phalanx.

Blazar et al. in their study on fractures of proximal interphalangeal joint stated that an understanding of the anatomy, the potential for joint instability and the treatment options are essential to manage these fractures [9]. They also described various treatment options including extension-block splinting, percutaneous pinning, traction, external fixation, open reduction and internal fixation and volar-plate arthroplasty. A prompt recognition of the complexity of the injury and appropriate management are also

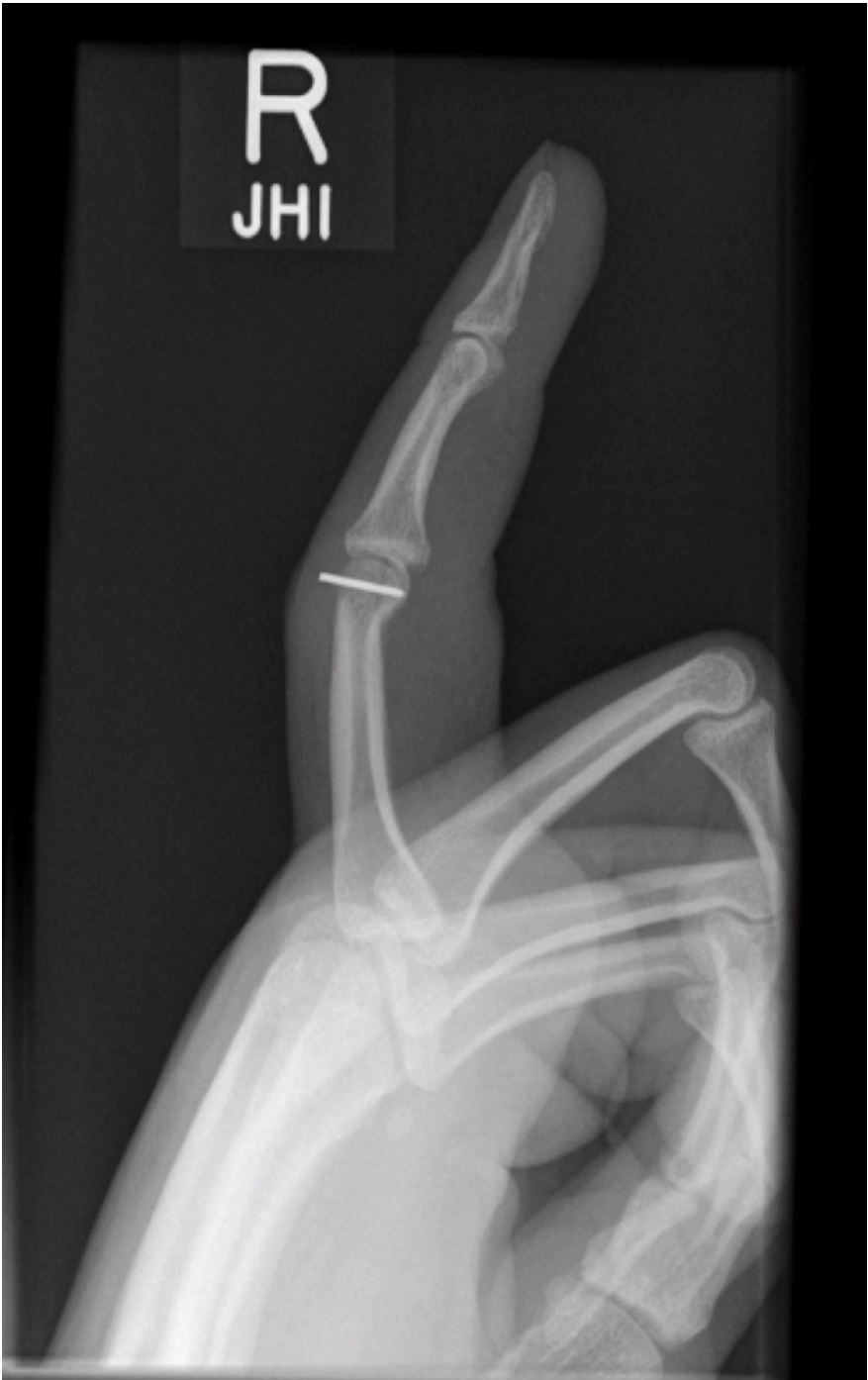

Figure 4: Check radiograph showing accurate reduction of fracture of head of the proximal phalanx.

essential for an optimal functional outcome.

The objectives of techniques of internal fixation of proximal phalangeal fractures are pain control and early functional restoration. When the fragment size permits, unstable and displaced proximal phalangeal joint fractures can be secured in an anatomic position by either a direct method through the fragments or by indirect methods (buttress) like pinning or screw fixation techniques. Percutaneous or limited open reduction and internal fixation techniques are preferred in an effort to minimize additional soft tissue trauma and scarring [10].

The classification of distal unicondylar fractures of the proximal phalanx was based on the mechanism of injury. There are four class of fractures [3]. The patient in our case had a class 4 fracture which involved a volar coronal fragment. These injuries are caused by a shearing force across an extended proximal interphalangeal joint causing a transient volar subluxation of the PIP joint with the joint extended. Class 4 fractures have the poorest final range of motion and this may be 


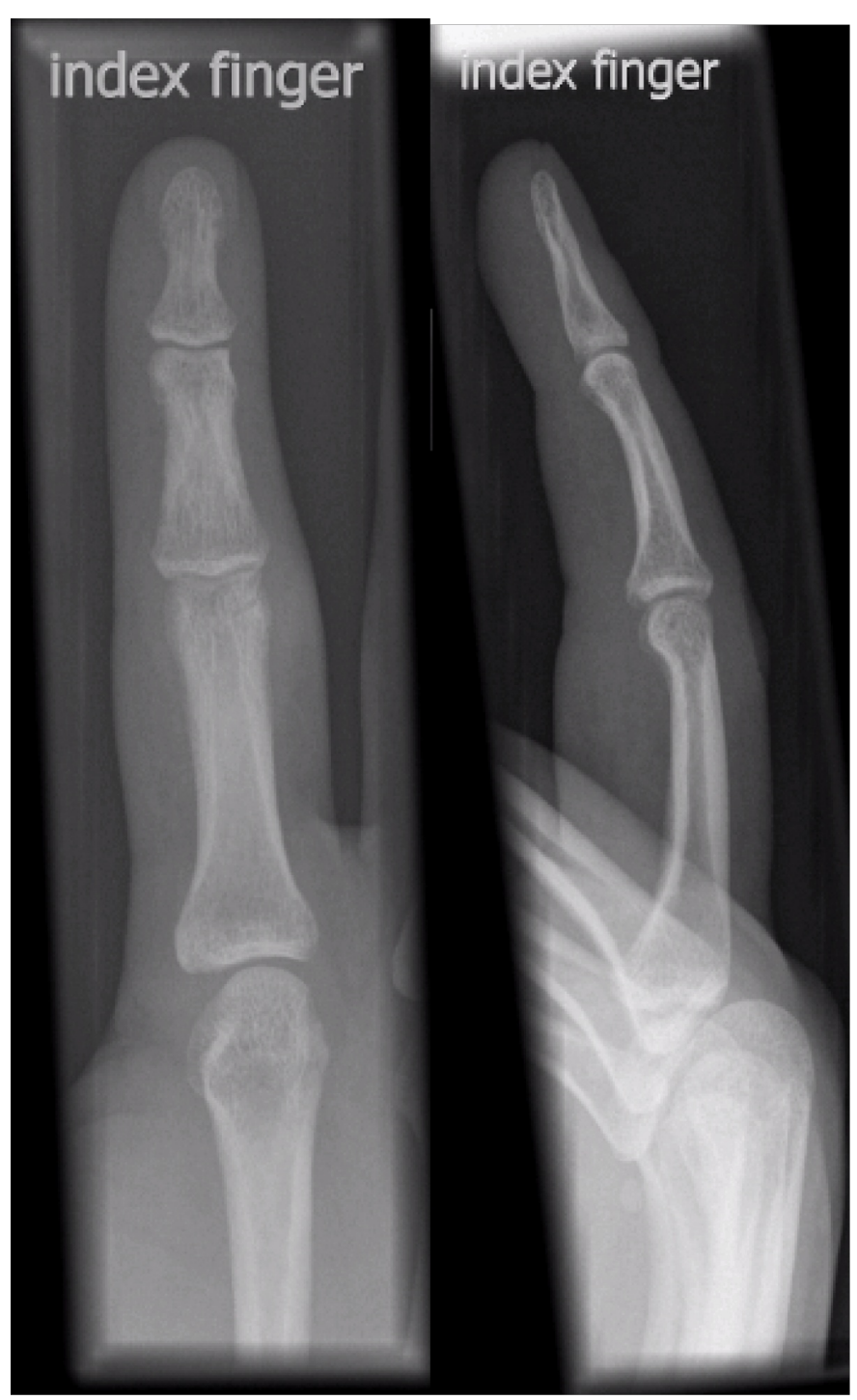

Figure 5: Radiographs showing a well healed fracture of head of proximal phalanx (unicondylar).

due to imperfect reduction.

\section{CONCLUSION}

Our technique of fixing unicondylar fractures of the proximal phalanx used a single Kirschner wire introduced from the volar aspect to engage the fracture fragment. We felt a screw placed from dorsal surface may not gain adequate fixation in the thin volar fragment and the prominent screw head may affect joint flexion, and if removal was necessary this would require more dissection than a subcutaneous Kirschner wire.

\section{Author Contributions}

Aysha Rajeev - Conception and design, Acquisition of data, Analysis and interpretation of data, Drafting the article, Critical revision of the article, Final approval of the version to be published

John Harrison - Conception and design, Acquisition of data, Analysis and interpretation of data, Drafting the article, Critical revision of the article, Final approval of the version to be published

\section{Guarantor}

The corresponding author is the guarantor of submission.

\section{Conflict of Interest}

Authors declare no conflict of interest.

\section{Copyright}

(C) Aysha Rajeev et al. 2013; This article is distributed under the terms of Creative Commons Attribution 3.0 License which permits unrestricted use, distribution and reproduction in any means provided the original authors and original publisher are properly credited. (Please see www.ijcasereportsandimages.com/copyright-policy.php for more information.)

\section{REFERENCES}

1. Glickel SZ, Barron OA. Proximal interphalangeal joint fracture dislocations. Hand Clin 2000;16(3):333-44.

2. Weiss AP, Hastings $\mathrm{H}$ 2nd. Distal Unicondylar Fractures the Proximal Phalanx. Journal of Hand Surgery Am 1993;18(4):594-9.

3. Hastings $\mathrm{H}$ 2nd, Carroll $\mathrm{C} 4$ th. Treatment of closed articular fractures of the metacarpophalangeal and proximal interphalangeal joints. Hand Clin 1988;4(3):503-27.

4. London PS. Sprains and fractures involving the interphalangeal joints. Hand 1971;3(2):155-8.

5. McCue FC, Honner R, Johnson MC, Gieck JH. Athletic injuries of the proximal interphalangeal joint requiring surgical treatment. J Bone Joint Surg 1970;52(5):937-56.

6. Soeur R. Fractures of the limbs: the relationship between mechanism and treatment. Brussels: La Clinique Orthopedique 1981:542-3.

7. Ramos LE, Becker GA, Grossman JA. A treatment approach for isolated unicondylar fracture of the proximal phalanx. Ann Chir Main Memb Super 1997;16(4):305-9.

8. Henry MH. Fractures of the proximal phalanx and metacarpals in the hand: preferred methods of stabilization. $\mathrm{J}$ Am Acad Orthop Surg 2008;16(10):586-95.

9. Blazar PE, Steiberg DR. Fractures of the proximal interphanlangeal joint. J Am Acad Orthop Surg 2000;8(6):383-90.

10. Freeland AE, Benoist LA. Open reduction and internal fixation method for fractures at the proximal onterphalangeal joint. Hand Clin 1994;10(2):239-50. 
Access full text article on other devices

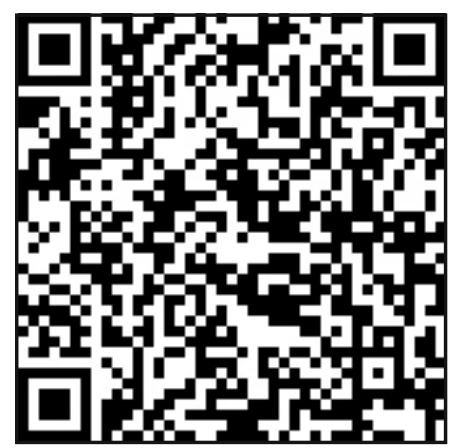

Access PDF of article on other devices

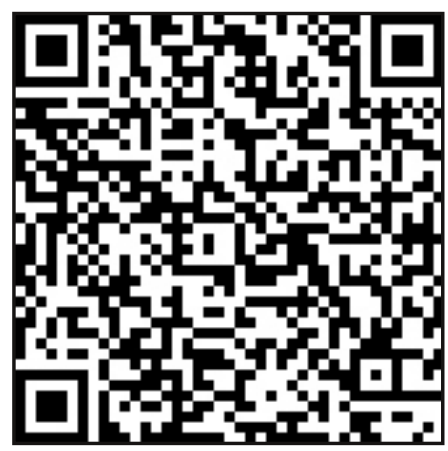

